\title{
Depinning with Dynamic Stress Overshoots: Mean Field Theory
}

\author{
J.M. Schwarz and Daniel S. Fisher \\ Lyman Laboratory of Physics, Harvard University, Cambridge, Massachusetts 02138
}

(December 13, 2000)

\begin{abstract}
An infinite-range model of an elastic manifold pulled through a random potential by an applied force $F$ is analyzed focusing on inertial effects. When the inertial parameter, $M$, is small, there is a continuous depinning transition from a small- $F$ static phase to a large- $F$ moving phase. When $M$ is increased to $M_{c}$, a novel tricritical point occurs. For $M>M_{c}$, the depinning transition becomes discontinuous with hysteresis. Yet, the distribution of discrete "avalanche"-like events as the force is increased in the static phase for $M>M_{c}$ has an unusual mixture of first-order-like and critical features.
\end{abstract}

PACS Numbers: 68.35.Rh, 83.50.Tq

A wide variety of driven systems are characterized by some type of elastic, extended object that is pulled through a quenched random medium by a uniform applied force. If the dynamics is dissipative- e.g. sliding charge density waves [1], vortex lattices in superconductors [2] and domain walls in ferromagnets [3] -such systems exhibit a critical depinning transition in the absence of thermal fluctuations. A small applied force, $F$, is not enough to overcome the random pinning forces and the system remains trapped in one of many possible metastable configurations. However, as $F$ is slowly increased, some sections become unstable and move, only to be stopped by the elastic forces from more strongly pinned neighboring regions. As $F$ is increased even further, there will be a sequence of these discrete, localized "avalanche" events with a distribution of sizes, $s$ [4]. As a critical force $F_{c}$ is approached from below, arbitrarily large avalanches can occur. Above $F_{c}$, the driving force is able to overcome the pinning, giving rise to a nonzero average velocity, $\bar{v}$. The transition between the two phases is continuous with $\bar{v}$ playing the role of an order parameter that vanishes as $\bar{v} \sim\left(F-F_{c}\right)^{\beta}$ as $F_{c}$ is approached from above. This class of dissipative systems has been analyzed by renormalization group (RG) methods [1] with the results supported by numerical [5] and experimental [6] evidence, although quantitative experimental tests have been very limited.

In some systems, in particular crack fronts in brittle materials [7] and motion of contact lines of droplets on dirty or rough solid surfaces [8], the dynamics are not dissipative and inertial effects can be important. In this Letter, we explore the effects of inertial stress transfer in which the motion of one segment creates a transient stress (in addition to the static elastic stress) on other segments. In the presence of pinning, the motion will be mostly forward; this makes the positive (forward pulling) parts of the transient stress transfer the most important; we call these stress overshoots. Although we will focus only on such dynamic stress transfer effects here, the more direct effects of inertia will have similar consequences: if a segment moves forward in an underdamped manner so that it overshoots - passing a new local minimum before relaxing back into it - the stress it transfers to the other regions will reflect this overshoot.

Our focus will be on the nature of the depinning transition in the presence of stress overshoots; a key question is whether or not the transition from the pinned to the moving phase remains continuous or becomes "firstorder" - characterized by a finite jump in $\bar{v}$ as a function of the applied force and perhaps by hysteresis. We will also consider the distribution of avalanche sizes as the depinning transition is approached from below.

Near the depinning transition, the motion will be very jerky with a lot of starting and stopping. Thus, the essential physics can be captured by models in which space, time and the manifold's displacement in the direction of motion, $h(\mathbf{x}, t)$, are all discrete. The elastic stress on a segment $\mathbf{x}$ takes the general form

$$
\sigma(\mathbf{x}, t)=\sum_{\mathbf{y}} \sum_{\tau \geq 0} J_{\mathbf{x y}}(\tau) h(\mathbf{y}, t-\tau)-\hat{J} h(\mathbf{x}, t),
$$

with the static stress transfer given by $J_{\mathbf{x y}}^{s}=\sum_{\tau} J_{\mathbf{x y}}(\tau)$ and $\hat{J}=\sum_{\mathbf{y}} J_{\mathbf{x y}}^{s}$. The simplest form of the stress overshoots is when they only last for one time step, i.e. $J_{\mathbf{x y}}(\tau \geq 2)=0$, and $J_{\mathbf{x y}}$ is non-zero only for nearest neighbors. In this case we write, $J_{\mathbf{x y}}(0)=\frac{(1+M)}{Z}$ and $J_{\mathbf{x y}}(1)=-\frac{M}{Z}$ for $\mathbf{x} \neq \mathbf{y}$ where $Z$ is the number of nearest neighbors and $M$ is the magnitude of the stress overshoot. With this dynamic stress transfer, the motion of any neighboring segment, $\mathbf{y}$, causes an extra stress on the $\mathbf{x} t h$ segment for one time step only. For $M<0$, a key property of the elastic stress transfer is that it is monotonic, i.e. increasing in time when the $h$ 's increase. This monotonicity property can be used to prove the uniqueness of the average velocity and thereby a unique critical force [9]. It is also an essential feature for the validity of the RG analysis [1]. For $M>0$, the stress transfer is non-monotonic in time so that the average velocity is not necessarily a unique function of $F$.

We take the displacements $h(\mathbf{x}, t)$ to be pinned at a discrete set of possible values with associated pinning (yield) 
strengths $f_{Y}[\mathbf{x}, h(\mathbf{x}, t)]$. The dynamics is simple: if the total force on segment $\mathbf{x}$ exceeds $f_{Y}$ at that point, the segment jumps forward, i.e. if $\sigma(\mathbf{x}, t)+F>f_{Y}[\mathbf{x}, h(\mathbf{x}, t)]$ then $h(\mathbf{x}, t+1)=h(\mathbf{x}, t)+\Delta[\mathbf{x}, h(\mathbf{x}, t)]$ with $\Delta$ the distance to the next pinning position; otherwise $h(\mathbf{x}, t+1)=$ $h(\mathbf{x}, t)$. As long as the jumps $\Delta[\mathbf{x}, h(\mathbf{x}, t)]$ are random variables independently drawn from a smooth distribution $D(\Delta) d \Delta$, the randomness in the pinning strengths is not essential and, for simplicity, we make them all equal.

We now take the first step in analyzing such systems by studying a mean field- more precisely an infinite-range -model. For the case without stress overshoots, such a model was the needed starting point for the RG analysis of finite-dimensional systems. We thus consider a model of $N$ segments each coupled to all the others. The stress on $h(x, t)$ is then simply

$$
\sigma(x, t)=\bar{h}(t)-h(x, t)+M(\bar{h}(t)-\bar{h}(t-1)),
$$

where $\bar{h}(t) \equiv \frac{1}{N} \sum_{y} h(y, t)$ is the average over the other segments.

In this infinite range model, all sites are statistically equivalent and coupled only via the mean field, $\bar{h}(t)$. We can therefore fully characterize the configuration by the distribution of the excess force on a segment $f_{t}(x) \equiv \sigma(x, t)+F-f_{Y}$. Dropping the $x$ index, we denote this distribution, $\rho_{t}\left(f_{t}\right) d f_{t}$. Since each segment is either pinned, $f_{t} \leq 0$, or will jump forward by some random amount, $\Delta$, the equation of motion for the excess force distribution is

$$
\begin{array}{r}
\rho_{t+1}\left(f_{t+1}\right)=\int_{-\infty}^{\infty} d \phi_{t} \delta\left(\phi_{t}+\bar{v}_{t+1}+M\left(\bar{v}_{t+1}-\bar{v}_{t}\right)-f_{t+1}\right) \\
\times\left\{\rho_{t}\left(\phi_{t}\right) \Theta\left(-\phi_{t}\right)+\int_{0}^{\infty} D(\Delta) \rho_{t}\left(\phi_{t}+\Delta\right) \Theta\left(\phi_{t}+\Delta\right) d \Delta\right\},
\end{array}
$$

where $\phi_{t}$ is the new excess force on a segment before the others have moved, $\bar{v}_{t} \equiv \bar{h}(t)-\bar{h}(t-1)$ and $\Theta$ is the unit step function. The self-consistency relation for $\bar{v}_{t}$ follows from the "spatial" averaging of the excess force:

$$
<f_{t}>=\int_{-\infty}^{\infty} f_{t} \rho_{t}\left(f_{t}\right) d f_{t}=F-f_{Y}+M \bar{v}_{t}
$$

For static solutions, $\bar{v}=0$, the stress overshoot plays no role and one can show that there are many solutions as long as the applied force is less than the static critical force $F_{c 0}=f_{Y}-\frac{1}{2} \frac{\overline{\Delta^{2}}}{\Delta}$, where the bars here denote averages over $D(\Delta)$. We will return later to the response to an increase in the force in this regime. For now, we turn to the moving phase.

We first consider the steady state limit for which $\bar{v}_{t}=\bar{v}>0$ is independent of time. Equation (4) implies that in this case the stress overshoot has the same effect as an additional applied force, $F \rightarrow F+M \bar{v}$, and the steady state $\rho(f)$ depends only on $\bar{v}$ (and $D$ ). It is easiest to work with the particular jump distribution
$D(\Delta)=e^{-\Delta}$ which corresponds to random pinning positions with density $1 / \bar{\Delta}$ chosen to be unity. However, the qualitative properties of the $\bar{v}(F)$ curve are applicable to any well-behaved, smooth pinning distribution with $D(0)$ finite and non-zero. For $M<1$ and $F>F_{c 0}, \bar{v}$ rises continuously as

$$
\bar{v} \sim \frac{F-F_{c 0}}{1-M}
$$

for $\bar{v}<<\bar{\Delta}=1$. The fact that $\bar{v}$ is linearly proportional to $F-F_{c}$ in mean field models with jumps has been derived previously for dissipation-dominated systems[1]; it is due to the fact that above the depinning transition the average displacement of a segment responds linearly in a non-singular way to an increase in the total force on it. Note that as the applied force is increased further, the $\bar{v}$ of the model is constrained in an unphysical way by the average distance between pinning sites. As a result, $\bar{v}$ will saturate in a manner that depends on the particulars of the pinning distribution. For the exponentially decaying jump distribution, the full expression for $\bar{v}$ is

$$
\bar{v}=\frac{-\epsilon_{0}+\mu+\sqrt{\left(\epsilon_{0}+\mu\right)^{2}+2 \epsilon_{0}}}{1+2 \mu}
$$

with $\epsilon_{o} \equiv F-F_{c 0}$ and $\mu \equiv M-M_{c}$.

As $M$ increases, the amount of applied force required to reach a specific $\bar{v}$ decreases. When $M$ reaches a critical value of $M_{c}=1$, the width of the continuous depinning transition shrinks to zero. When both the parameters $M$ and $F$ are critical there is a "tricritical" point. Near this tricritical point the scaling behavior is $\bar{v} \sim|\mu| \sim \sqrt{\epsilon_{0}}$, indicating a new universality class of depinning transitions.

For $M>M_{c}$, there is a jump in $\bar{v}$ from $\bar{v}_{\text {min }} \sim \mu$ to zero when $F$ is decreased through a new lower critical force $F_{c}^{\downarrow}=F_{c 0}-(1+\mu-\sqrt{1+2 \mu})$. The two stable solutions in this regime consist of $\bar{v}=0$ and a branch with $\frac{d \bar{v}}{d F}<0$. There is also a third solution, but it is unstable. Between $F_{c}^{\downarrow}$ and $F_{c 0}$ there is hysteresis as might be expected at a first-order like phase transition. A greater decrease in the force is required to stop the motion in the presence of overshoots than in their absence, but once the overall motion does stop the overshoots will have less effect as $F$ is increased back up again.

We now investigate the avalanche dynamics below the depinning threshold. An avalanche is the forward motion of a finite number of segments in response to the initial motion of one segment. The applied force is increased only in order to trigger the motion of the initial segment and is then held fixed until the avalanche stops. The segments that move forward in a single finite avalanche are those whose $f$ is within an infinitesimal- $\mathcal{O}\left(\frac{1}{N}\right)$ - slice near $f=0$ of the distribution $\rho(f)$; for continuous $\rho(f)$ all that matters is thus $\rho(0)$. The total number of segments that hop forward at one time step, $n_{t}$, will have poissonian statistics whose mean is determined by the increase in the total force on a segment from the previous 
time step. The mean number that hop at a given time $t$ is given by

$$
<n_{t}>=\rho(0) \bar{\Delta}\left[(1+M) n_{t-1}-M n_{t-2}\right] .
$$

If this mean becomes negative, the avalanche will stop. We define the parameter, $\Gamma \equiv \rho(0) \bar{\Delta}$, which is essentially the mean local response to an increase in the total force.

We are primarily interested in the large avalanches, for which $n_{t}$ will typically be large. Given large $n_{t-2}$ and $n_{t-1}$, the randomness will cause approximately gaussian fluctuations in $n_{t}$ of magnitude of order $\sqrt{n_{t}}$. With no stress overshoots, $M=0$, we can approximate the dynamics in continuous time in the Ito representation as

$$
\frac{d n(t)}{d t} \simeq(\Gamma-1) n(t)+\sqrt{n(t)} \eta(t)
$$

with $\eta$ white noise. The constraint that $n(t)$ be positive makes finding the distribution of the avalanche size, $s \equiv \int d t n_{t}$, a first passage problem. A critical point occurs at $\Gamma=1\left(\rho_{c}(0)=\frac{1}{\Delta}\right)$, at which there is a power law distribution of avalanche sizes, $\operatorname{Prob}(d s) \sim \frac{1}{s^{\frac{3}{2}}} d s$ for large $s$ [10]. Beyond this point, infinite avalanches occur as the motion of one segment spawns more than one descendant at the next time step. For smaller $\Gamma$, a cutoff in the avalanche size distribution given by $\tilde{s} \sim \frac{1}{(1-\Gamma)^{2}}($ which is not equal to the average as the distribution is very broad), and $\operatorname{Prob}(d s) \sim \frac{1}{s^{\frac{3}{2}}} e^{-\frac{(1-\Gamma)^{2} s}{4}} d s$ for large $s$ as shown in Fig. 1.

In the more general case with stress overshoots, the stochastic equation becomes second-order in time since the number of segments that hop forward is dependent on two previous time steps and we have, approximately,

$$
\begin{array}{r}
\Gamma M \frac{d^{2} n(t)}{d t^{2}} \simeq(\Gamma-1) n(t)+(\Gamma M-1) \frac{d n(t)}{d t} \\
+\sqrt{n(t)} \eta(t) .
\end{array}
$$

When we introduce a small amount of stress overshoot, the avalanche dynamics is strongly overdamped. The critical point is still at $\Gamma=1$ but the distribution of avalanche sizes is modified by the coefficient $(M-1)$.

When the stress overshoot magnitude is increased to the critical value, $M_{c}=1$, the avalanche dynamics becomes undamped resulting in a new exponent for the distribution of avalanche sizes. At this tricritical point, we have $\frac{d^{2} n(t)}{d t^{2}}=\sqrt{n(t)} \eta(t)$ which maps the distribution of avalanche sizes into a first passage problem of a particle with random acceleration. The distribution of avalanche sizes broadens to $\operatorname{Prob}(d s) \sim \frac{1}{s^{\frac{5}{4}}} d s$ for large $s$ [11]. The typical avalanche of size $s$ now lasts for a time $\tau \sim s^{\frac{1}{4}}$ in contrast to $\tau \sim s^{\frac{1}{2}}$ in the absence of stress overshoots. The scaling form of the distribution within the vicinity of this tricritical point is

$$
\operatorname{Prob}(d s) \sim \frac{1}{s^{5 / 4}} F\left(s\left(M-M_{c}\right), s^{1 / 2}(1-\Gamma)\right) d s .
$$

For $M>M_{c}$, the deterministic part of the avalanche equation of motion is that of a time-reversed harmonic oscillator. If $\rho(0)$ is small-i.e., below the critically damped line $-n(t)$ grows exponentially with time before crashing down to zero as a result of the oscillation. Above some critical $\Gamma, \Gamma_{c}(M)=\frac{4 M}{(1+M)^{2}}$ (from Eq. (7)), large avalanches grow exponentially and become infinite. On the critical line, some avalanches become infinite while others remain finite. Because of the exponential growth of $n(t)$ both above and below the critical line, the randomness will not have much of an effect once an avalanche becomes large and a deterministic analysis where all the randomness is encoded into random initial $n$ and $\frac{d n}{d t}$ becomes valid. Just below the critical line, there is a clear delineation in the distribution of avalanche sizes between those avalanches that would remain finite and those that would become infinite at the critical line. Indeed, just below $\Gamma_{c}(M)$, a finite fraction of the avalanches will have size of order $\hat{s} \sim \exp \left(A(M) / \sqrt{\left(\Gamma_{c}(M)-\Gamma\right)}\right)$ to within a multiplicative factor of order unity; these last roughly half the oscillation period. Note that for $M \gtrsim 1$, $A \sim \mu \equiv M-1$ and the scaling of $1-\Gamma$ versus $\mu$ is like that of $F-F_{c 0}$ versus $\mu$ in the moving phase. Most of the other avalanches will be small, but with a long tail in $\log s$ scaling as

$$
\operatorname{Prob}(d s) \sim \frac{1}{s(\ln s)^{2}} d s=\frac{d(\ln s)}{(\ln s)^{2}}
$$

for $1<<\ln s<<\ln \hat{s}$. This is an unusual distribution with $\ln s$ being the natural variable. This broad distribution for the finite avalanches persists at the depinning transition, but now there is also a non-zero probability that an avalanche will be infinite as indicated in Fig. 1. These results suggest a new hybrid transition for $M>M_{c}$ with a diverging scale, $\hat{s}$, but also discontinuities, particularly in $\bar{v}(F)$.

So far, we have not established the connection between the driving force, $F$, and the density of about-to-jump segments $\rho(0)$. This is needed to connect together the analysis of the pinned and the moving phases. As long as the avalanches remain finite and the driving force is adiabatically increased, the total force distribution $\rho(f) d f$ will be independent of $M$; the avalanche distribution will only affect when segments jump and hence the small scale $\left(f \sim \frac{1}{N}\right)$ behavior of the stationary configurations between avalanches. For generic initial conditions - continuous $\rho(f)$ which must be consistent with randomly positioned pinning values of $h(x, t)-\Gamma$ will increase with $F$ to reach unity only right at $F_{c 0}$, although the way in which it approaches this value is non-universal and history dependent. Thus for $M<1, \Gamma_{c}=1$ and the infinite avalanches will only occur when $F>F_{c 0}$, the same point above which $\bar{v}>0$. 


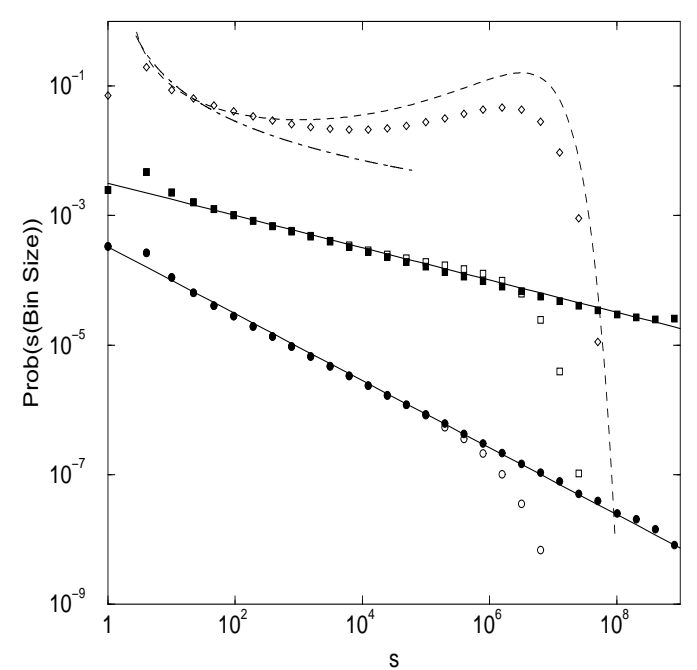

FIG. 1. Log-log plot of the probability of an avalanche occurring of size within the interval $[s / \sqrt{2}, \sqrt{2} s)$. The circles are numerical data in the absence of stress overshoots, with solid circles at the critical $\Gamma=1$ and open circles just below, $\Gamma=0.999$. The squares are at the critical $M=M_{c}=1$. The closed squares are at the tricritical point $\Gamma=1$ with the open squares just below, $\Gamma=0.999$. The solid lines have slope $\frac{1}{2}$ and $\frac{1}{4}$, representing theoretical predictions for the critical $M<1$ and tricritical $M=1$ points. The open diamonds are for $M=2$ and $\Gamma=0.88$, just below the "first-order" line; these should roughly match the dashed curve which is from the deterministic approximation with an exponential distribution of initial velocities. The dot-dashed curve represents Eq. (11).

For $M>1$, however, $\Gamma$ will reach the value $\Gamma_{c}(M)$ at a value of the driving force $F_{c}^{\uparrow}$, that depends on the initial conditions as well as on $M$. From an analysis of $\mathrm{Eq}(3)$, it can be shown that $F_{c}^{\uparrow}$ is between $F_{c}^{\downarrow}(M)$, the minimum $F$ for which a moving solution exists, and $F_{c 0}$, as should be expected. Thus, if $F$ is increased above $F_{c}^{\uparrow}$ and back down there will be hysteresis. If $F$ is decreased below $F_{c}^{\downarrow}$ so that the system stops and is then increased back up again, the behavior can be complicated as $\rho(f)$ will generally not be continuous. Depending on the parameters (including $D(\Delta)$ ) and the history, the depinning transition on the second increase of $F$ can either be of the hybrid type discussed above with an exponentially growing characteristic size, $\hat{s}$, of large avalanches which diverges at a new $F_{c}^{\uparrow}$; or a strongly discontinuous transition with $\Gamma$ jumping from below $\Gamma_{c}$ to above discontinuously and no extensive number of precusor large avalanches.

We have found that in a simple infinite-range model of depinning with dynamic stress overshoots, rather subtle behavior can occur. For large overshoots, a hybrid transition exists when the force is increased adiabatically. This is characterized by exponentially-large, almost-deterministic avalanches followed by runaway at a critical force to a non-zero velocity state; this moving state persists to a lower critical force as the force is decreased back down. Various history dependent effects can occur in this large overshoot regime. For smaller stress overshoots, there is a reversible critical transition similar to that in the absence of overshoots. These two regimes are separated by a "tricritical" point which represents a new universality class.

All of this behavior should obtain for a broad class of infinite-range models, including ones with locally underdamped relaxation caused by inertia $[12,13]$. But the crucial question is: what of this behavior persists in finitedimensional models? For large stress overshoots the depinning is surely of a different character than in purely dissipative systems, although what aspects of it might resemble the infinite-range model is far from clear. For small stress overshoots, it has been argued elsewhere that the critical force on increasing $M$ is generically less than $F_{c 0}$ in finite-dimensional models with long-range interactions [14]. This is the relevant situation for fronts of planar cracks propagating through heterogeneous brittle materials and one would guess that the same should hold more generally. But whether this implies that the transition immediately changes character when any stress overshoots are present - and thus presumably that there is no tricritical point - or whether dissipative-like critical behavior can still exist with small overshoots, we must leave as an open question.

The authors would like to thank Ron Maimon and Cristina Marchetti for useful discussions. This work has been supported in part by the National Science Foundation via DMR-9630064, DMR-9976621, and DMR9809363.

1 O. Narayan and D. S. Fisher, Phys. Rev. B 46, 11520 (1992).

2 G. Blatter et al., Rev. Mod. Phys. 66, 1125 (1994).

3 K. A. Dahmen and James P. Sethna, Phys. Rev. B 53, 14872 (1996).

4 P. Bak, C. Tang, K. Wiesenfeld, Phys. Rev. Lett. 59, 381 (1987).

5 A. A. Middleton and D. S. Fisher, Phys. Rev. Lett. 66, 92 (1991); O. Narayan and A. A. Middleton, Phys. Rev. B 49, 244 (1994).

6 M. J. Higgins, A. A. Middleton and S. Bhattacharya, Phys. Rev. Lett. 70, 3784 (1993).

7 S. Ramanathan, D. Ertas and D. S. Fisher, Phys. Rev. Lett. 79, 873 (1997).

8 D. Ertas and M. Kardar, Phys. Rev. Lett. 69, 929 (1992).

9 A. A. Middleton, Phys. Rev. Lett. 68670 (1992).

10 see e.g. D. S. Fisher, Phys. Reps., 301, 113 (1998).

11 Y. G. Sinai, Theor. Math. Phys. 90, 219 (1992); J. M. Schwarz and Ron Maimon (unpublished).

12 D. A. Head and G.J. Rodgers, Phys. Rev. E 55, 2573 (1996).

13 M. C. Marchetti, A. A. Middleton and T. Prellberg, Phys. Rev. Lett. 85, 1104 (2000).

14 S. Ramanathan and D. S. Fisher, Phys. Rev. B 58, 6026 (1997). 